\title{
A New Neural Pathway from the Ventral Striatum to the Nucleus Basalis of Meynert with Functional Implication to Learning and Memory
}

\author{
Si Yun Shu ${ }^{1}$. Gang Jiang ${ }^{2}$. Zhaocong Zheng ${ }^{3} \cdot$ Lin Ma $^{4} \cdot$ Bin Wang ${ }^{1}$. Qiyi Zeng ${ }^{1} \cdot$ Hong Li $^{1} \cdot$ Shen Tan ${ }^{5} \cdot$ Bin Liu $^{6}$. \\ Wood Yee Chan ${ }^{7}$. Sheng Wu ${ }^{8}$. Chunhua Zhu ${ }^{8} \cdot$ Changke $\mathrm{Li}^{9} \cdot$ Peng Wang ${ }^{10} \cdot$ Jang-Yen $\mathrm{Wu}^{11}$ (D)
}

Received: 31 October 2018 / Accepted: 27 March 2019 / Published online: 18 April 2019

(C) The Author(s) 2019

\begin{abstract}
The cholinergic neurons in the nucleus basalis of Meynert (NBM) are among the first group of neurons known to become degenerated in Alzheimer's disease, and thus the NBM is proposed to be involved in learning and memory. The marginal division (MrD) of the striatum is a newly discovered subdivision at the ventromedial border of the mammalian striatum and is considered to be one part of the ventral striatum involved in learning and memory. The present study provided evidence to support the hypothesis that the MrD and the NBM were structurally connected at cellular and subcellular levels with functional implications in learning and memory. First, when wheat germ agglutinin-conjugated horseradish peroxidase (WGA-HRP) was stereotaxically injected into the NBM, fusiform neurons in the MrD were retrogradely labeled with WGA-HRP gray-blue particles and some of them were double stained in brown color by AchE staining method. Thus, cholinergic neurons of the MrD were shown to project to the neurons in the NBM. Second, in anterograde tract-tracing experiments where WGA-HRP was injected to the MrD, the labeled WGA-HRP was found to be anterogradely transported in axons from the $\mathrm{MrD}$ to the synaptic terminals with dendrites, axons, and perikaryons of the cholinergic neurons in the NBM when observed under an electronic microscope, indicating reciprocal structural connections between the MrD and the NBM. Third, when bilateral lesions of the MrD were injured with kainic acid in rats, degenerative terminals were observed in synapses of the NBM by an electronic microscope and severe learning and memory deficiency was found in these rats by the Y-maze behavioral test. Our results suggest reciprocal cholinergic connections between the MrD of the ventral striatum and the NBM, and implicate a role of the MrD-NBM pathway in learning and memory. The efferent fibers of cholinergic neurons in the NBM mainly project to the cortex, and severe reduction of the cholinergic innervation in the cortex is the common feature of Alzheimer's patients. The newly discovered cholinergic neural pathway between the $\mathrm{MrD}$ of the ventral striatum and the NBM is supposed involved in the memory circuitries of the brain and probably might play a role in the pathogenesis of the Alzheimer's disease.
\end{abstract}

Si Yun Shu

shusiyun@126.com

Jang-Yen Wu

jwu@health.fau.edu

Gang Jiang

jiangali6370@163.com

Zhaocong Zheng

zhengzhaocong@126.com

Lin Ma

cjr.malin@vip.163.com

Bin Wang

wangbin6556@126.com

Qiyi Zeng

zqy_88@163.com

Hong Li

13380091998@126.com
Shen Tan

tansheng18@126.com

Bin Liu

nysylb@163.com

Wood Yee Chan

wy-chan@cuhk.edu.hk

Sheng Wu

13588196809@139.com

Chunhua Zhu

hzlyyyk@163.com

Changke Li

anesthesia@126.com

Peng Wang

13688860780@163.com

Extended author information available on the last page of the article 
Keywords The striatum - The nucleus basalis of Meynert (NBM) - Learning and memory $\cdot$ Tract tracing $\cdot$ Y-maze Immunocytochemistry $\cdot$ Electronic microscopy $\cdot$ Alzheimer's disease

\section{Introduction}

The subcortical striatum is a heterogeneous structure including the ventral striatum (nucleus accumbens), the dorsolateral striatum, and the dorsomedial striatum, and is believed to be involved in modulation of complex motional [1] and learningmemory activities [2]. The ventral striatum (nucleus accumbens) is known to play a predominant role in association with reward and motivation [3], whereas the dorsolateral striatum is able to formulate stimulus-response strategies such as habit formation [4], body-centered memory including egocentric coordination [5], and unconscious procedural memories that are strengthened during trial-and-error learning [6]. The dorsomedial striatum, similar to the caudate nucleus in humans, is associated with goal-directed learning [7]. Neuropathological evidence indicates decreases of cholinergic interneurons with reduced choline acetyltransferase (ChAT) activities in the striatum of patients with Alzheimer's disease [8]. Some recent studies have provided behavioral and anatomical evidence to support the notion that associations exist between different brain regions including the striatum and the hippocampus [9-11], the striatum and the prefrontal cortex [12], the striatum and the thalamus [13], the striatum and the amygdala [14], the hippocampus and the prefrontal cortex [15], the hippocampus and the thalamus [16], the prefrontal cortex and the thalamus [17], the hippocampus and the amygdala [18], the nucleus basalis of Meynert (NBM) and the medial temporal lobe system [19], and the amygdala and the thalamus [20], but observations on the direct anatomical connection between the striatum and the NBM are still absent [21,22].

Previous studies identified a new brain area, the marginal division $(\mathrm{MrD})$, at the ventromedial border of the striatum in the rat, cat, monkey, and human [23-25]. The MrD is distinguished from the rest of the striatum by its spindle-shaped neurons, specific connections, and dense immunoreactivities of neuropeptides and monoamines in fibers, terminals, and neuronal somata [23-26] (Fig. 1). The 5'-nucleotidase activity is densely expressed in the developing rodent $\mathrm{MrD}$ [27], whereas the a2-adrenergic receptors are expressed more strongly in the $\mathrm{MrD}$ than in the rest of the rat striatum [28]. Furthermore, it has been shown that the pedunculopontine nucleus sends to massive afferents to the $\mathrm{MrD}$ of the squirrel monkey [29], and that the $\mathrm{MrD}$ is connected to the interstitial nucleus of the posterior limb of the anterior commissure [30]. The MrD has been suggested to be one of the five components of the ventral striatum [31]. Behavioral tests and physiological experiments showed that the MrD contributes to learning and memory or pain reception in the rat $[32,33]$. Reduction in the capacity of learning and memory was observed in the Y-maze test after chemically induced bilateral lesions of the MrD of the rat [32]. The c-Fos protein was expressed in the hippocampus, dentate gyrus, amygdala, and the forebrain cortex after injection of kainic acid into the $\mathrm{MrD}$, demonstrating the functional connections between the $\mathrm{MrD}$ and these structures [34]. In addition, the $\mathrm{MrD}$ was found to have fiber connections with the amygdaloid nucleus [35] and the bed nucleus of the striatum terminals [36]. Moreover, it was also found that the efferent fibers from the MrD projected to the most caudal part of the globus pallidus, where the NBM was located. In the human brain, the association of the MrD with learning and memory has been demonstrated with both pathological case reports and functional MRI analyses [25], thus leading to the suggestion that the MrD of the neostriatum is a subcortical memory center and a new component of the limbic system [37]. Substance $\mathrm{P}$ which was expressed in the $\mathrm{MrD}$ was found to play a role in learning and memory and mediated through the neurokinin 1 receptor in rats [38]. Interactions between the hippocampus and the striatum were reported during episodic encoding [39]. Immunohistochemical study found that the distribution of muopioid receptor in the $\mathrm{MrD}$ was different from those in other parts of the neostriatum [40]. Different expression patterns of microRNAs were also observed in the $\mathrm{MrD}$ and the hippocampus of the rat [41]. Previous studies suggested that the $\mathrm{MrD}$ and the hippocampus may play different roles in learning and memory through different neurotransmission mechanism [42]. New functional and structural pathways related to learning and memory through the hippocampus, the amygdala, and the MrD in rats have been demonstrated [43].

Unlike the aforementioned relations between the striatum and various brain structures, less attention has been paid to the connection between the striatum and the NBM. The NBM as the major source of cholinergic innervation of the cerebral neocortex belongs to the basal forebrain cholinergic system [44]. As the degeneration of the cholinergic projections from the basal forebrain to the neocortex and the hippocampus has been found to be correlated with memory decline in Alzheimer's disease [45], it is proposed that the striatum may also be associated with the NBM during the process of learning and memory [46]. Although cognitive deficits are usually considered to be attributed by the loss or degeneration of cholinergic neurons in the NBM [46, 47], some reports have cast doubt on this proposition [48, 49]. Hence, both associations between the striatum and the NBM and the cognitive deficits and loss of synapses in the NBM need to be further examined. 


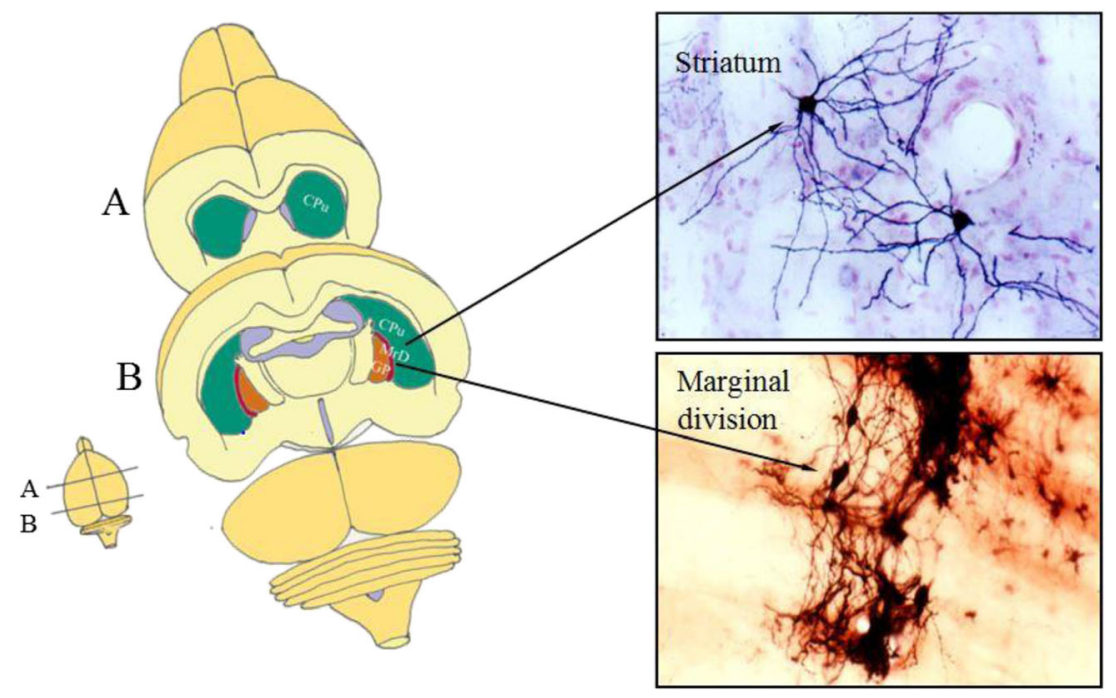

Fig. 1 Location and cytoarchitectural characteristics of the MrD in the ventral portion of the striatum in the rat brain. The diagram on the left side indicates two frontal sections (A and B) of the rat brain. The upper right panel shows that the PHA-L-labeled neuronal bodies in the dorsal part of the striatum (caudoputamen, $\mathrm{CPu}$ ) are mostly of round or triangular shape. The lower right panel illustrates the MrD located in the

In the present study, we carefully dissected detailed anatomical connections between the MrD and the NBM with wheat germ agglutinin-conjugated horseradish peroxidase (WGAHRP) tract-tracing, histochemical staining, electronic microscopy, and immunoelectronic microscopic techniques. The mechanism underlying the learning-memory process associated with the connections between the $\mathrm{MrD}$ and the NBM was also demonstrated through lesions of the MrD followed by studies in the Y-maze behavioral test with tract-tracing, electronic microscopy, and immunoelectronic microscopic techniques.

\section{Materials and Methods}

\section{Animals}

A total of 100 male Sprague-Dawley rats weighing 200-250 g were obtained from Beijing Research Center for Experiment Animals, Beijing, China, and used in this study. The rats were housed individually at a constant temperature of $25^{\circ} \mathrm{C}$ with ad libitum access to food and water in a 12:12-h light/dark cycle. They were randomly divided into two large groups: behavioral test group $(n=80)$ and non-behavioral test group $(n=40)$. Rats in the non-behavioral test group were randomly divided into four subgroups: (1) control group with no treatment $(n=5)$; (2) WGA-HRP-treated group with $1 \%$ WGA-HRP injected into the MrD or NBM ( $n=15, n=15)$; (3) kainic acid (KA)-treated group with $0.1 \% \mathrm{KA}$ injected directly into the $\operatorname{MrD}(n=10)$; and (4) normal saline (NS)-treated group with NS injected into the $\operatorname{MrD}(n=10)$. For the behavioral test group, after the pretest in the Y-maze (see below), rats $(n=53)$ were randomly ventromedial portion of the striatum $(\mathrm{CPu})$ and dorsolateral to the globus pallidus (GP). The MrD consists of a band of fusiform neurons labeled by PHA-L, which distinguishes the MrD from the rest of the striatum $(\mathrm{CPu})$ and the GP. $\mathrm{Cpu}$, caudoputamen of the striatum corpus; GP, globus pallidus; $\mathrm{MrD}$, marginal division of the striatum

divided into five subgroups: (1) Bilaterally lesioned MrD group ( $n=18)$; (2) unilaterally lesioned MrD group ( $n=10)$; (3) bilaterally lesioned caudoputamen $(\mathrm{CPu})$ group $(n=10)$; (4) bilaterally lesioned NBM group $(n=10)$ and (5) bilaterally salineinjected $\mathrm{MrD}$ group $(n=5)$. All experimental procedures on animals were approved by the Institute for Animal Care of the Southern Medical University. All efforts were made to minimize the number of animals used and any pain they might experience during the course of the investigation.

\section{Histochemical and Tract-Tracing Methods}

One percent WGA-HRP (Sigma) $(0.2 \mu \mathrm{l})$ was stereotaxically injected into the NBM or the MrD of the rat's brain through a micropipette as the retrograde and anterograde tracers in the non-behavioral test group. Two days after WGA-HRP injections, the rats in the non-behavorial test group were anesthetized with $10 \%$ chloral hydrate $(3.5 \mathrm{ml} / \mathrm{kg}$, intraperitoneal (i.p.)). Under deep anesthesia, the rats were perfused through the aorta with $200 \mathrm{ml} 0.9 \%$ saline followed by $200 \mathrm{ml}$ fixative solution containing $3 \%$ paraformaldehyde and $0.25 \%$ glutaraldehyde in $0.02 \mathrm{M}$ sodium phosphate buffer $\left(\mathrm{pH} 7.4,4^{\circ} \mathrm{C}\right)$ within $1.5 \mathrm{~h}$. After fixation by perfusion, the brains were removed and immersed in the same fixative solution for $4 \mathrm{~h}$ before they were transferred to and immersed in a phosphate-buffered $20 \%$ sucrose $(0.02 \mathrm{M}$ sodium phosphate buffer, $\mathrm{pH} 7.4,4^{\circ} \mathrm{C}$ ) until the brains sank to the bottom of the solution. Brains were sectioned coronally on a vibratome (LKB, Sweden) at thickness of $50 \mu \mathrm{m}$. The sections were immersed in $0.01 \mathrm{M}$ phosphate buffer $\left(\mathrm{pH} \mathrm{7.4,4}{ }^{\circ} \mathrm{C}\right)$ for Nissl staining and acetylcholine esterase (AchE) staining or 
AchE-TMB (3,3', 5, 5'-tetramethylbenzidine) double staining. The WGA-HRP was visualized after reacting with 3,3',5, 5'tetramethylbenzidine (TMB) (Sigma) according to the method described by Mesulam [50]. The sections were prepared and examined with a light microscope (Olympus).

Nissl staining was carried out with $0.1 \%$ cresyl violet for examination of the Nissl bodies and morphology of neuronal bodies in the brain. Cryostat sections were mounted on slides and stained with $0.1 \%$ cresyl violet, then dehydrated, cleared, and coverslipped. AchE staining was carried out as follows [51]: after five changes of acetate buffer (1 min each), sections were treated with $1 \%$ ammonium sulfide solution for $1 \mathrm{~min}$ followed by five changes of $0.1 \mathrm{M}$ sodium nitrate ( $1 \mathrm{~min}$ each). Sections were then exposed to $0.1 \%$ silver nitrate for $1 \mathrm{~min}$ followed by five changes of $0.1 \mathrm{M}$ sodium nitrate ( $1 \mathrm{~min}$ each). Free-floating sections were rinsed in the acetate buffer, mounted from acetate buffer onto subbed slides, air-dried, dehydrated, cleared, and coverslipped. Sections were counterstained before dehydration. AchE-TMB double staining was carried out as follows [52]: after incubation in AchE staining solution for $60 \mathrm{~min}$, sections were rinsed in $\mathrm{dH}_{2} \mathrm{O}$ for six times ( 1 min each). Then they were reacted with $1 \%$ ethylene diamine tetraacetic acid (EDTA) solution for $1 \mathrm{~min}$, and further incubated in $1 \%$ $\mathrm{H}_{2} \mathrm{O}_{2}$ solution for $20 \mathrm{~min}$, and then glucose saline (GS, $40 \mathrm{mg} /$ $100 \mathrm{ml}$ ) and glucose oxidase (GOD, $1 \mathrm{mg} / 100 \mathrm{ml}$ ) were added to the incubation solution for an additional $20 \mathrm{~min}$. The color reaction was visualized using $10 \%$ potassium ferricyanide after the sections were rinsed in the acetate buffer for six times (30 min each). After color development, the sections were airdried, dehydrated, cleared, and coverslipped.

\section{Lesion}

All rats were anesthetized with an i.p. injection of $10 \%$ chloral hydrate $(3.5 \mathrm{ml} / \mathrm{kg})$. After anesthetized, they were subsequently placed on a stereotaxic platform. Their scalps were opened longitudinally with a scalpel and three small holes were drilled in the skull at the coordinates indicated in the bregma system [53]. Glass micropipettes were introduced through the small holes into the brain to perform microinjection of KA, WGA-HRP, or saline $(0.2 \mu \mathrm{l}$ each). The coordinates were $1.4 \mathrm{~mm}$ posterior to the bregma, 4.2, 5.0, or $3.2 \mathrm{~mm}$ lateral to the midline, and 5.3, 5.0, or $6.6 \mathrm{~mm}$ ventral from the skull surface for the $\mathrm{MrD}, \mathrm{CPu}$, or NBM injection, respectively. After each injection, the needle was left in place for $10 \mathrm{~min}$ before slowly retracted.

\section{Behavioral Test}

An electric Y-maze was chosen. This Y-maze uses light as the conditional visual stimulus combined with the avoidance of electric foot-shock pain reinforcement to test associative learning and declarative memory, commonly known as foot shockmotivated brightness discriminating Y-maze test [54]. The Y- maze has three arms with metal wires on their bottom to deliver electric shocks and lights at their ends. When the foot-shock avoidance test began, one arm with the light on (light zone) was the shock-free area, whereas the other two arms with the light off (dark zone) were areas with electric shocks. Electric shocks were delivered to any of these three arms during the test. Rats preferred to enter the dark zones at the beginning of the test. After receiving a foot shock, most of the rats soon learned to escape from the dark to the light zone to avoid electric shocks. During the test, the rats were considered to be able to learn and remember the correct route of escape from the electric shock if they could run to the light zone within $10 \mathrm{~s}$ after the light shifted from one to another arm of the Y-maze. The number of correct escapes (running to the light zone within $10 \mathrm{~s}$ ) in 30 electric shocks was used to quantify memory. All rats $(n=80)$ were tested twice before carrying out the behavioral tests and only those who passed the first test with at least 10 correct escapes and passed the second test with at least 15 correct escapes were used in the subsequent behavioral tests. Two days later after passing the two preliminary tests, the rats in the above groups were tested in the foot-shock avoidance Y-maze with simultaneous changes in the light stimulus. After this avoidance test, the rats who were able to learn that the light end was safe were chosen $(n=53)$ and divided randomly into five subgroups as indicated in the "Animals" section. The rats were then stereotaxically injected with $0.1 \% \mathrm{KA}(0.2 \mu \mathrm{l})$ into the MrD bilaterally or unilaterally, the CPu bilaterally, or the NBM bilaterally to produce the specific lesions. Saline was injected bilaterally into the MrD of the control animals. The avoidance tests were carried out again with the Y-maze 2 days after the lesion.

\section{Electron Microscopic Examinations}

Small pieces of the NBM were cut from the AchEhistochemically stained brain sections and WGA-HRPstained brain sections under a microscope. After postfixed in $2.5 \%$ glutaraldehyde for $2 \mathrm{~h}$, the tissues were washed in phosphate buffer and transferred into $0.5 \%$ osmium tetroxide at $4{ }^{\circ} \mathrm{C}$ for $1 \mathrm{~h}$, and then rinsed in distilled water and dehydrated through a graded series of ethanol. The tissues were incubated in $1 \%$ uranyl acetate dissolved in $70 \%$ ethanol, processed with propylene oxide, embedded, and then mounted. Ultrathin sections were cut on a LKB-Nova Ultratome (Sweden), stained with $1 \%$ lead citrate, and examined under a JEM-1200 (Japan) transmission electron microscope.

\section{Data Analysis}

Statistical analyses were performed with SPSS 17.0 software by SPSS, Inc. All data were presented as means \pm SD. Multiple samples were compared using analysis of variance (ANOVA). Multiple means were assessed statistically with paired-samples $t$ test. Significant differences were set at $p<0.05$. 


\section{Results}

\section{Efferent Projections from the MrD to the NBM Shown by WGA-HRP Retrograde Tracing Method}

WGA-HRP technique is a valuable tract-tracing reagent for studying the nervous connections in the central nervous system. The WGA-HRP could be transported within nerve fibers both anteriorly and retrogradely. TMB staining is a very sensitive method to show the transported WGA-HRP. The WGAHRP was stained in gray-blue color particles within the plasma of the neuronal cell body or in the nervous fiber terminals by TMB staining. We used the WGA-HRP to trace the connection between the MrD and NBM. Following the stereotaxic injection of WGA-HRP into the NBM, little WGA-HRP detected as the gray-blue substance was observed at or near the injection site (Fig. 2a), whereas the retrogradely transported WGA-HRP which appeared as gray-blue particles was found prominently in some fusiform neurons in the MrD (Fig. 2b) suggesting that WGA-HRP present at the MrD site is not due to non-specific uptake of the injected WGA-HRP tracer but due to a direct projection pathway between NBM and MrD. The retrogradely transported WGA-HRP-labeled fusiform neurons were observed in all five rats. There are eight retrogradely transported WGA-HRP-labeled neurons in the $\mathrm{MrD}$ of the striatum in the boxed area in one rat as shown in Fig. 3. The retrogradely transported WGA-HRP-labeled neurons in brain sections of the other four rats were $12,9,7$, or 10 ,

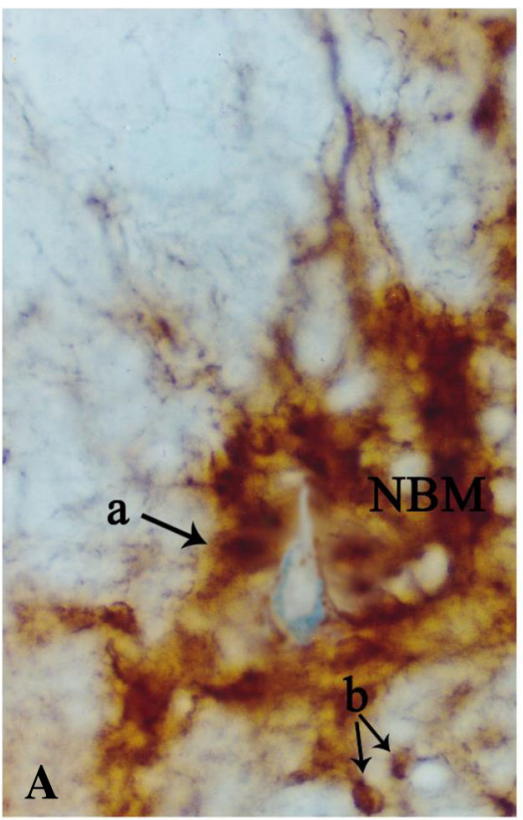

Fig. 2 Determination of the projection from the MrD to the NBM by WGA-HRP retrograde tracing method. a The WGA-HRP injecting site in the NBM is marked (arrow a) where little WGA-HRP tracer shown as blue substance could be detected. Two cholinergic neurons of NBM were observed at the right lower corner (arrow b). b WGA-HRP stained as gray-blue particles retrogradely labeled fusiform somata of neurons in respectively. Thus, the mean \pm SD of WGA-HRP-labeled neurons is $9.2 \pm 1.92$. Some of WGA-HRP-labeled neurons are also labeled with AchE. The ratio of AchE-WGA-HRP double-labeled neurons to Total WGA-HRP-labeled neurons was $0.500,0.417,0.444,0.429$, and 0.500 with the mean \pm SD as mean $0.458 \pm 0.040$ (Table 1). A dot plot showing the ratio as percentage of AchE-WGA-HRP double-labeled neurons to Total WGA-HRP-labeled neurons is shown in Fig. 4. The result showed definitely the presence of efferent projections from the MrD to the NBM. The brown substrate was stained by AchE method to demonstrate the cholinergic containing neurons and nerve fibers. The results demonstrated that the $\mathrm{MrD}$ contains cholinergic neurons and some of these cholinergic neurons projected to NBM.

\section{Loss of Fusiform Neurons in the MrD After KA Injection Shown by Nissl Staining}

The KA is a toxin to neurons in the brain. We stereotaxically injected $0.1 \% \mathrm{KA}(0.2 \mu \mathrm{l})$ into the MrD bilaterally or unilaterally, the $\mathrm{CPu}$ bilaterally, or the NBM bilaterally to produce the specific lesions. Only 5 out of $15 \mathrm{KA}$-injected rats were injected right in bilateral $\mathrm{MrD}$ and 8 of 15 rats were injected in NBM precisely. The saline as a control reagent was injected bilaterally into the $\mathrm{MrD}$ in the control animals. The fusiform neurons which were parallel in the ventromedial border of the striatum were lost and were replaced with a large number of infiltrated microglia in the sections through the KA injection

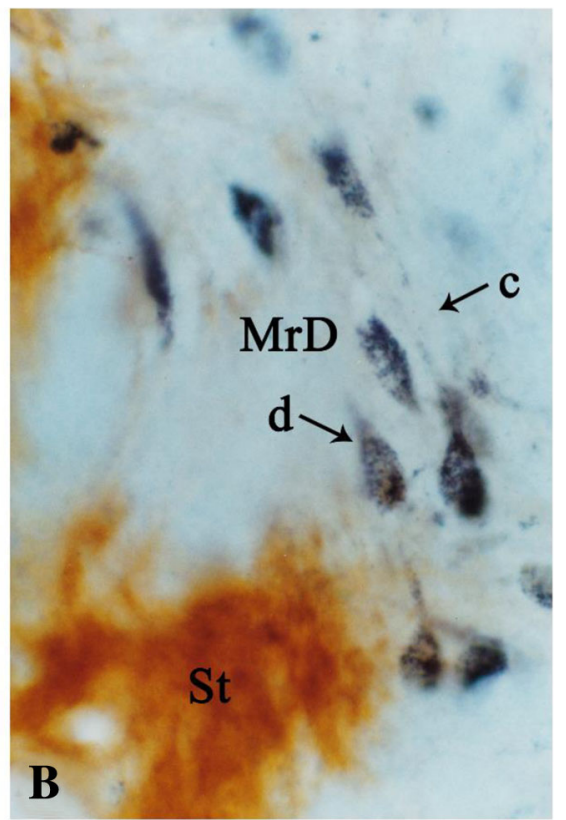

the $\mathrm{MrD}$ of the ventral striatum (arrow c) in the rat brain. Some of the WGA-HRP-labeled neurons were stained with brown color background, which were AchE-WGA-HRP double-labeled neurons (arrow d). The AchE-WGA-HRP double-labeled neurons were cholinergic neurons in $\mathrm{MrD}$, which projected to the $\mathrm{NBM}$. MrD, marginal division of the striatum; NBM, basal nucleus of Meynert. Magnification $=\times 400$ 


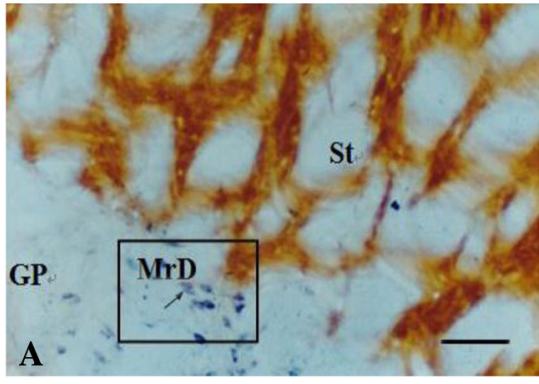

Fig. 3 The projection from the MrD to the NBM by WGA-HRP retrograde tracing method. The conditions were the same as in Fig. 2. a WGA-HRP stained as gray-blue particles retrogradely labeled fusiform somata of neurons (arrows) in the MrD of the ventral striatum in the rat brain. b High magnification of the boxed area in a. Some fusiform

site in the $\mathrm{MrD}$, while the morphology of neurons in the adjacent striatum and globus pallidus was still normal (Fig. 5). The neurons of the $\mathrm{CPu}$ and the NBM were lost as well in the KA-injected group. No obvious histopathological changes were found in the sections of the rats brains in the salineinjected group. The results indicate that the KA injections had injured the neurons in the $\mathrm{MrD}$ or $\mathrm{CPu}$ or $\mathrm{NBM}$ of the rat brains, respectively.

\section{Identification of AchE-Containing Neurons and Substrate in the MrD, Striatum, and NBM}

The cholinergic neurons in sections of the brain were stained in brown or dark brown color by the AchE method. The dorsal and lateral striatum in the rat brain was heavily stained in brown color fully by AchE staining method of the salineinjected group, and the staining was so heavy that individual neurons were not easily discernible (Fig. 6). Some large multiangular cholinergic neurons in the NBM were also heavily stained with AchE method. A band of medium-size AchE positives-stained cholinergic fusiform neurons was observed in the $\mathrm{MrD}$ with their long axes arranged in parallel to the ventromedial border of the striatum (Fig. 6). The results indicated that both $\mathrm{MrD}$ of the striatum and NBM contain cholinergic neurons and nervous fibers.

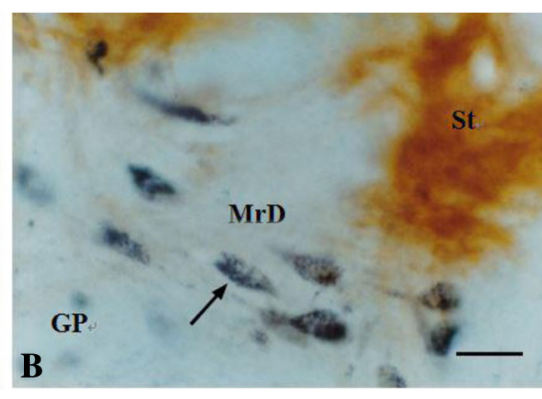

neurons were double-stained in brown color with some blue particles in their cell bodies. This AchE-WGA-HRP double-stained neurons were cholinergic neurons in the MrD which project to NBM. GP, globus pallidus; $\mathrm{MrD}$, marginal division of the striatum; St, striatum; scale bar $=400 \mu \mathrm{m}(\mathbf{a})$ and $100 \mu \mathrm{m}(\mathbf{b})$

\section{Demonstration of Degenerated Terminals in the NBM Identified by the Electron Microscope After KA Injected into the MrD}

The small pieces of the NBM were cut from sections of the $\mathrm{KA}$-injured $\mathrm{MrD}$ of the rat brain to investigate the degenerated terminal in the synapses of the NBM. These small pieces of tissues were incubated in $1 \%$ uranyl acetate dissolved in $70 \%$ ethanol, processed with propylene oxide, embedded, and then mounted. The embedded and mounted tissues were cut as ultrathin sections by a LKB-Nova Ultratome (Sweden). The ultrathin sections were examined under a JEM-1200 (Japan) transmission electron microscope for investigating the degenerating terminals projected from the MrD synapsed on the AchE-positive neurons of NBM. Under the transmission electron microscope, iron sulfide products with high electron densities from the AchE staining reaction were found scattered in the neurons, suggesting that these neurons were cholinergic neurons. Some axon terminals which showed substances with high electron densities and round synaptic vesicles were observed in the synapses of AchE neurons in the NBM. The degenerated terminals from the MrD which exhibited decreased numbers of synaptic vesicles, increased electron densities, and mitochondrial deformation or aggregation were observed in the synapses of the NBM (Fig. 7). These terminals formed the axo-somatic, axo-dendritic, axo-axonic,

Table 1 Ratio of AchE-WGA-HRP double-labeled neurons to Total WGA-HRP-labeled neurons in the MrD of the striatum. Ratio of AchE-WGAHRP double-labeled neurons to Total WGA-HRP-labeled neurons in percent

\begin{tabular}{lll}
\hline $\begin{array}{l}\text { Total WGA-HRP-labeled neurons in the box } \\
\text { Number of animals: }\end{array}$ & $\begin{array}{l}\text { AchE-WGA-HRP } \\
\text { double-labeled neurons }\end{array}$ & $\begin{array}{l}\text { Ratio of AchE-WGA-HRP double-labeled } \\
\text { neurons to Total WGA-HRP-labeled neurons (\%) }\end{array}$ \\
\hline Animal 1-8 & 4 & 50.0 \\
Animal 2-12 & 5 & 41.7 \\
Animal 3-9 & 4 & 44.4 \\
Animal 4-7 & 3 & 42.9 \\
Animal 5-10 & 5 & 50.0 \\
\hline
\end{tabular}

The mean $\pm \mathrm{SD}$ as mean $0.458 \pm 0.040$ 


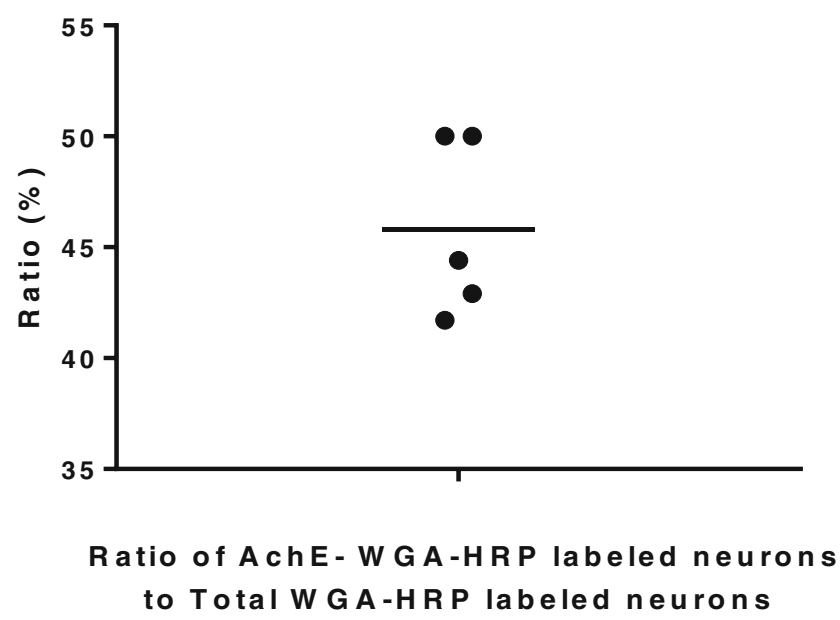

Fig. 4 A dot plot showing the ratio as percentage of AchE-WGA-HRP double-labeled neurons to Total WGA-HRP-labeled neurons

or complex synapses with cholinergic neuronal bodies, dendrites, and axons. Since these degenerated terminals were observed in the NBM after injection of $\mathrm{KA}$ to the $\mathrm{MrD}$, this observation suggested that the degenerated terminals were projected from the KA damaged $\mathrm{MrD}$ to the NBM.

\section{Demonstration of WGA-HRP Anterogradely Labeled Neural Terminals from MrD to NBM by Electron Microscopy}

The WGA-HRP could be transport within nerve fibers both anteriorly and retrogradely. TMB staining is a very sensitive method to show the transported WGA-HRP. The WGA-HRP was stained in gray-blue color particles within the plasma of the neuronal cell body or in the nervous fiber terminals. The WGA-HRP anterogradely transported in the terminals of NBM from the axons of MrD. The TMB-stained WGAHRP displayed as dark rod-like crystals in the labeled axonal

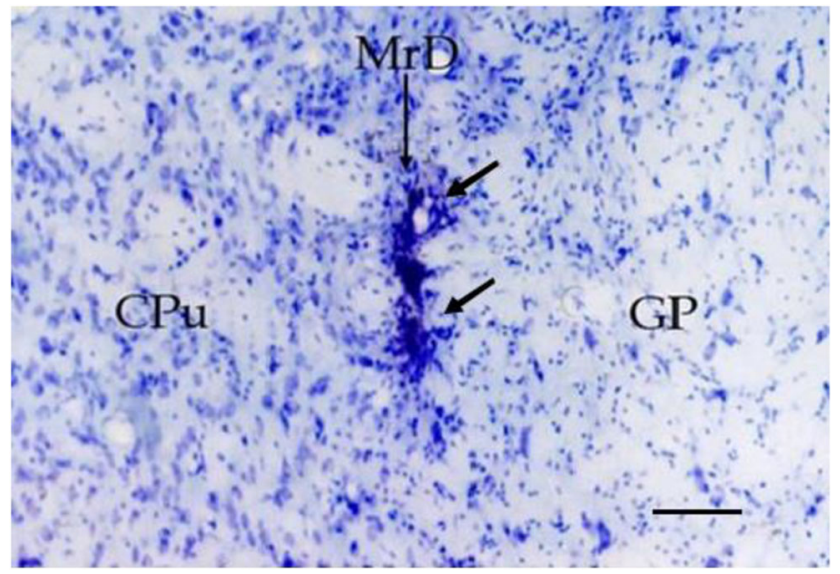

Fig. 5 Loss of fusiform neurons in the MrD after KA injection shown by Nissl staining. At the injection site, fusiform neurons in the $\mathrm{MrD}$ (upper longer arrow) were lost (pointed by two shorter parallel arrows), while the morphology of neurons in the adjacent striatum (caudoputamen, $\mathrm{CPu}$ ) and globus pallidus (GP) is still normal. Scale bar $=100 \mu \mathrm{m}$

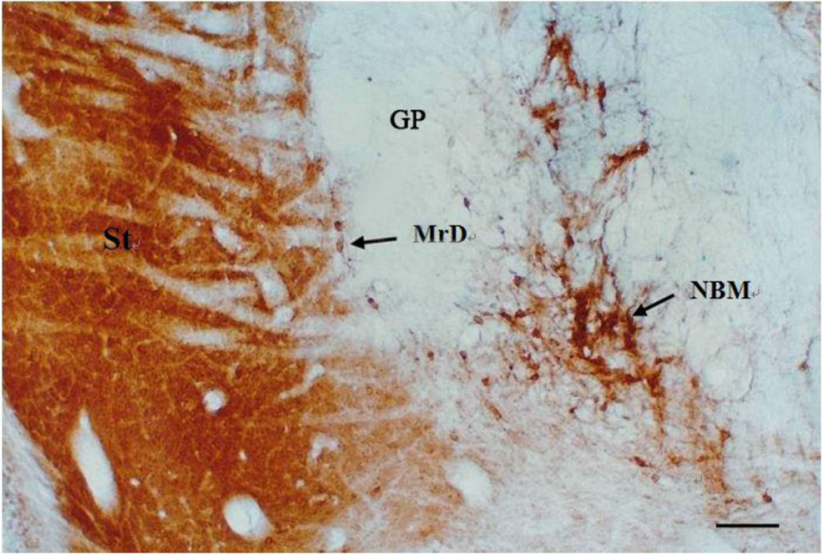

Fig. 6 Identification of AchE-containing neurons and neuronal processes in the $\mathrm{MrD}$, striatum, and the NBM. The cholinergic neurons and nerve fibers were stained in dark brown color by AchE staining method. A row of cholinergic medium-size fusiform neurons stained by AchE method in brown color was observed in the $\mathrm{MrD}$ of the striatum (black arrow on the left). The large multi-angular cholinergic neurons in the NBM were heavily stained by AchE method in brown color (black arrow on the right). The rest portion of the striatum (St) is fully filled with AchEpositive substrates. MrD, marginal division of the striatum; NBM, nucleus basalis of Meynert; St, striatum; scale bar $=400 \mu \mathrm{m}$

terminals under the transmission electron microscope, which were observed in synapses of the NBM after WGA-HRP was injected into $\mathrm{MrD}$. These anterogradely labeled neural terminals formed symmetric axo-somatic synapses with cholinergic neurons of the NBM under the electron microscope (Fig. 8).

\section{Behavioral Tests}

We used an electric Y-maze to test the behavioral ability of the rat. This Y-maze uses light as the conditional visual stimulus combined with the avoidance of electric foot-shock pain reinforcement to test associative learning and declarative memory, commonly known as foot shock-motivated brightness discriminating Y-maze test [54]. The light-foot shock avoidance reaction is based on the memory-related conditional reflex, and used to test the memory function of the animal. The Ymaze has three arms with metal wires on their bottom to deliver electric shocks and lights at their ends. When the footshock avoidance test began, one arm with the light on (light zone) was the shock-free area, whereas the other two arms with the light off (dark zone) were areas with electric shocks. Electric shocks were delivered to any of these three arms during the test. Rats all preferred to enter the dark zones at the beginning of the test. After receiving a foot shock, most of the rats soon learned to escape from the dark to the light zone to avoid electric shocks. After several shocks, rats learned and remembered that the light zone was a safe area and hence they ran directly to the light zone whenever the light was shifted from one area to the other. During the test, the rats were considered to be able to learn and remember the correct route of 


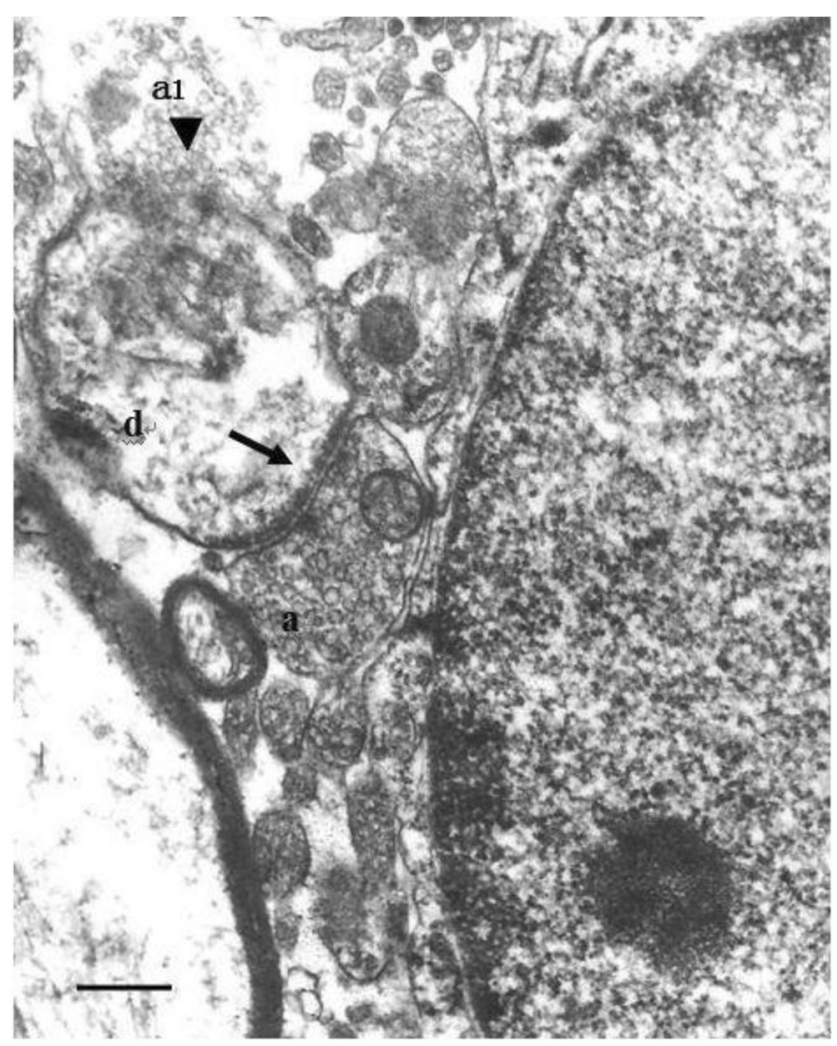

Fig. 7 Demonstration of degenerated terminals in the NBM after KA injection into the $\mathrm{MrD}$ - electron microscopic analysis. Anterograde degenerative axon terminals are observed in the NBM following KA injection to the $\mathrm{MrD}$ of the rat brain. A terminal (a) with synaptic vesicles and swollen mitochondria is found to form an asymmetric axodendritic synapse (arrow) on a dendrite (d). A completely damaged axon (a1, black triangle) with decreased numbers of synaptic vesicles and a disrupted axon membrane is found just above the dendrite (d). A cholinergic neuron with a big nucleus in the NBM is occupied the right panel of the figure. $\mathrm{MrD}$, marginal division of the striatum; $\mathrm{NBM}$, nucleus basalis of Meynert; scale bar $=0.5 \mu \mathrm{m}$

escape from the electric shock if they could run to the light zone within $10 \mathrm{~s}$ after the light shifted from one to another arm of the Y-maze. The number of correct escapes (running to the light zone within $10 \mathrm{~s}$ ) in 30 electric shocks was used to quantify memory. The behavioral test was performed with the Ymaze in the preliminary tests and also 2 days after the treatment with KA. The learning and memory scores of the rats were expressed by the total numbers of shocks required for rats to choose correctly 9 out of 10 times. No significant differences in the learning and memory scores among rats of different treatment groups were observed before the treatment $(p>0.05)$. The total number of shocks required for the $90 \%$ positive response was increased significantly after bilateral KA lesions of the $\operatorname{MrD}(n=12)$ or NBM $(n=6)(p<0.05)$, from $15.58 \pm 2.02$ to $28.58 \pm 2.02$ or $15.00 \pm 1.41$ to $26.83 \pm$ 1.33 , respectively (Table 2). No significant differences were found between these two groups $(p>0.05)$. Furthermore, no differences in the number of total shocks required for the $90 \%$ positive response before and after lesions in the groups of

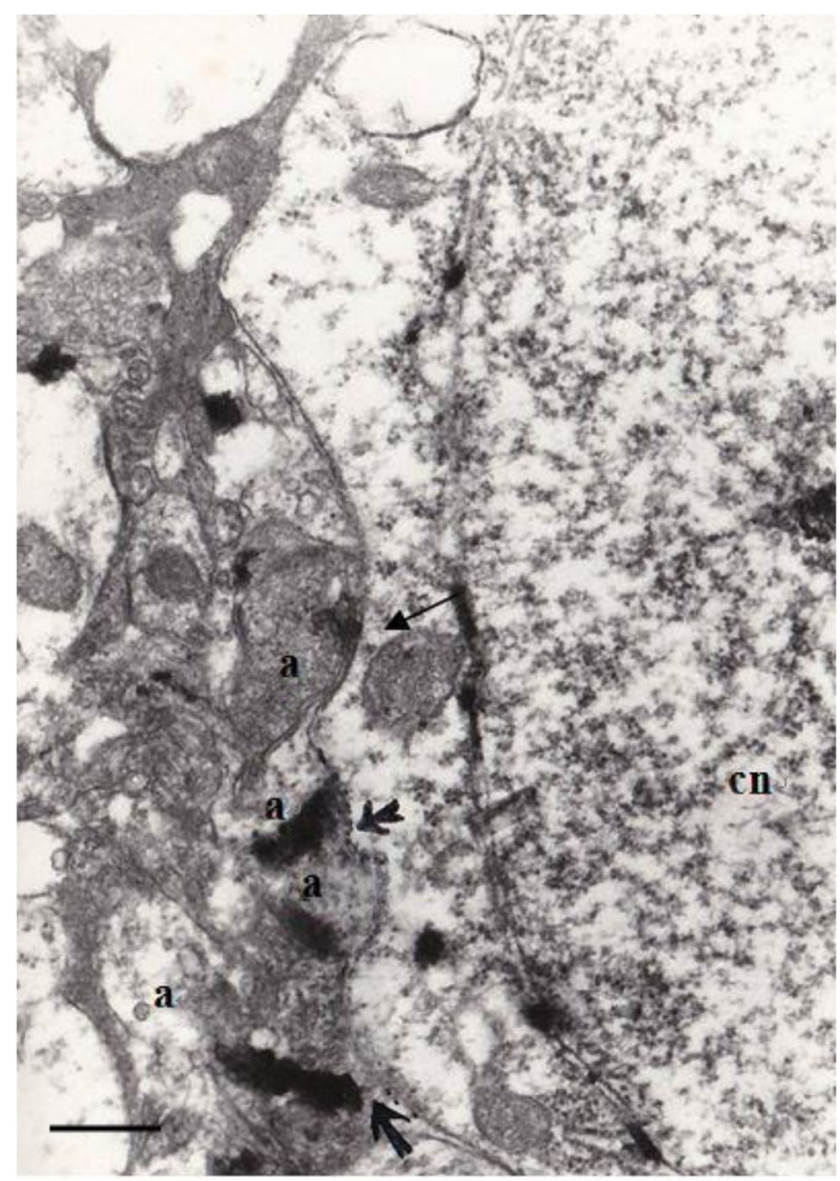

Fig. 8 Electron photomicrography showing anterograde WGA-HRPlabeled terminals projected from the MrD to the NBM after WGA-HRP injection in to the MrD. Following WGA-HRP injection to the MrD, anterogradely transported WGA-HRP, which appeared as rod-like electron dense substances, labeled axon terminals (a) from the MrD to form symmetric axo-somatic synapses (arrows) with a cholinergic neuron (cn) of the NBM. MrD, marginal division of the striatum; NBM, nucleus basalis of Meynert; scale bar $=0.5 \mu \mathrm{m}$

bilateral KA lesions of the caudoputamen (neostriatum) or unilateral $\mathrm{KA}$ lesions of the $\mathrm{MrD}$ as observed in the salineinjected control group $(p>0.05)$ (Table 2). These results suggested that bilateral lesions of the MrD or NBM led to the decrease of learning and memory ability.

\section{Discussion}

The results of the present study demonstrated that the $\mathrm{MrD}$ and the NBM were structurally connected at both cellular and subcellular levels using a combination of histochemical localization, tract-tracing, electron microscopy, and immunoelectronic microscopy techniques. WGA-HRP was employed to identify the direct connections between neurons, because it could be transported both anterogradely and retrogradely between the neuronal somata and their terminals. Following WGA-HRP injection to the NBM, positive gray-blue particles of WGA- 
Table 2 Total number of shocks required for attaining a $90 \%$ correct rate in the Y-maze test before and after the KA treatment

Total number of shocks ${ }^{\mathrm{a}}$

\begin{tabular}{llllll}
\hline Group & $N$ & Before treatment & & After treatment & $p$ value \\
\hline Bi-MrD lesion & 12 & $15.58 \pm 2.02$ & & $28.58 \pm 2.02$ & $<0.05$ \\
MrD lesion & 7 & $15.08 \pm 1.29$ & & $16.57 \pm 0.98$ & $>0.05$ \\
Bi-CPu lesion & 8 & $15.88 \pm 1.25$ & & $16.25 \pm 1.39$ & $>0.05$ \\
Bi-NBM lesion & 6 & $15.00 \pm 1.41$ & & $26.83 \pm 1.33$ & $<0.05$ \\
Control & 4 & $15.75 \pm 1.26$ & & $16.00 \pm 1.15$ & $>0.05$
\end{tabular}

${ }^{\text {a }}$ Values are expressed as mean $\pm \mathrm{SD}$

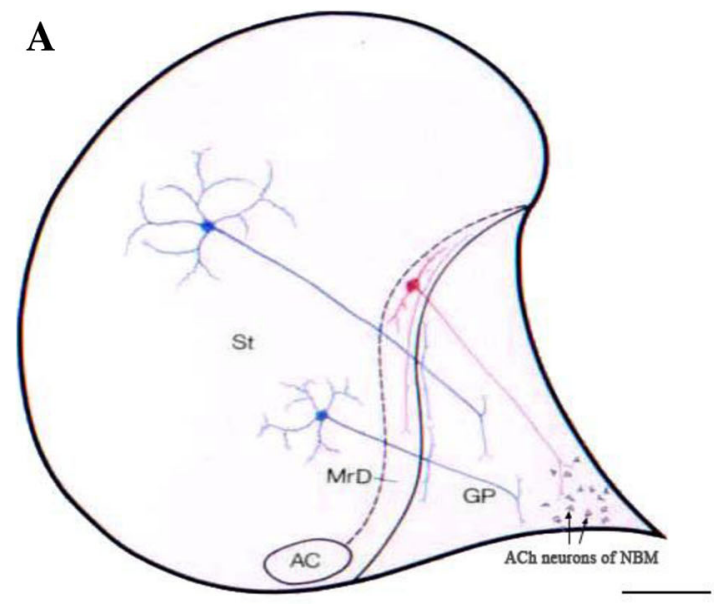

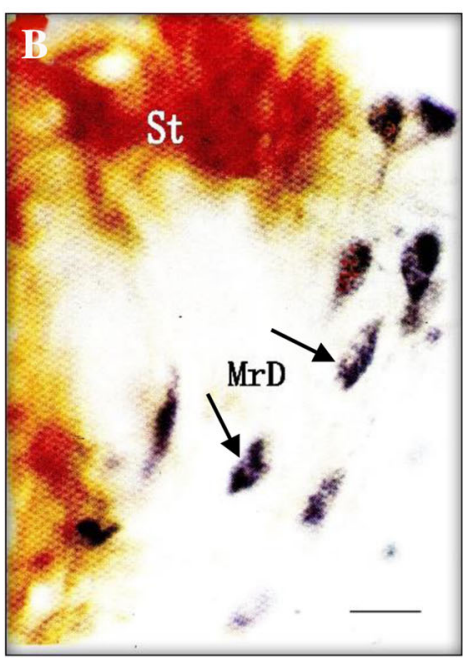
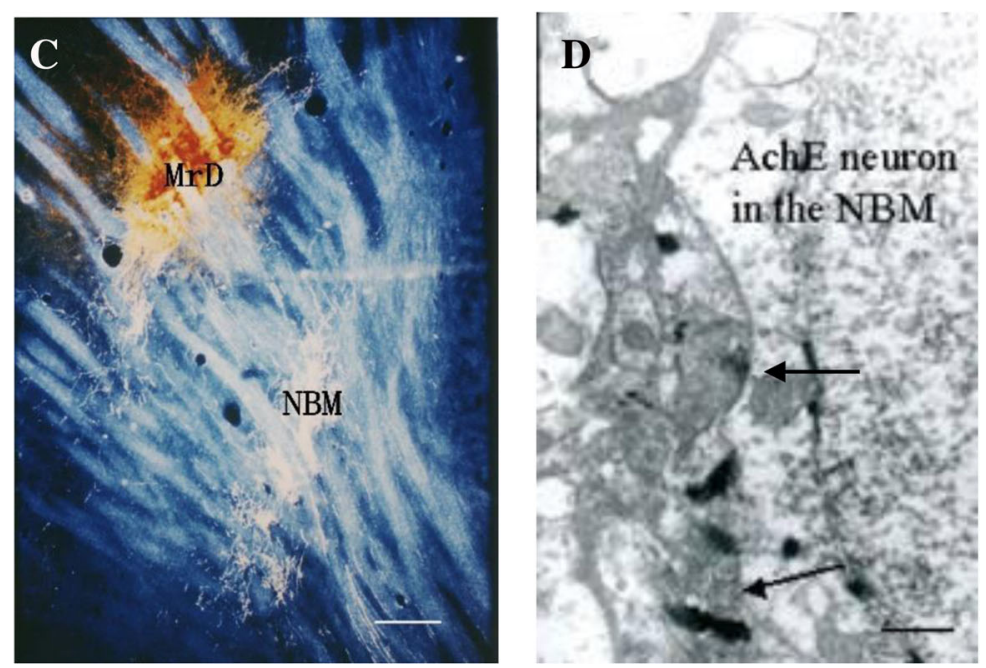

Fig. 9 Summary of the neuronal connections from the MrD to the NBM using the WGA-HRP tracing method. Schematic summary of the connection from the fusiform neuron (red) in the marginal division $(\mathrm{MrD})$ of the ventral striatum to the nucleus basalis of Meynert (NBM). The axons of round-shaped neurons (blue) in the other parts of the striatum (St) project to the globus pallidus (GP). b Following WGAHRP injection to the NBM, retrogradely transported WGA-HRP, which are stained as black-blue particles, labeled some fusiform neurons in the $\mathrm{MrD}$ of the ventral striatum. $\mathrm{MrD}$, marginal division of the striatum; NBM, nucleus basalis of Meynert. Scale bar $=100 \mu \mathrm{m}$. $\mathbf{c}$ The efferent projections (white lines) from the MrD to the NBM are observed in a dark field under a light microscope at low magnification. MrD, marginal division of the striatum; NBM, nucleus basalis of Meynert. Scale bar = $800 \mu \mathrm{m}$. d Following WGA-HRP injection to the MrD, anterogradely transported WGA-HRP, which appeared as rod-like electron dense substances in axon terminals from the $\mathrm{MrD}$, were found in the axonsomatic synapses(arrows) of cholinergic neurons in the NBM. MrD, marginal division of the striatum; NBM, nucleus basalis of Meynert. Scare bar $=1 \mu \mathrm{m}$
HRP were retrogradely transported within fusiform neurons in the $\mathrm{MrD}$, demonstrating efferent projections from the $\mathrm{MrD}$ to the NBM. Conversely, after WGA-HRP injection to the MrD, WGA-HRP labeling of axon terminals was found in the synapses of cholinergic neurons in the NBM under an electron microscope, lending support to the notion that the $\mathrm{MrD}$ and the NBM were reciprocally connected.

The Y-maze test has been widely used for identification of discrimination learning, spatial alternation tasks, and working and reference memory $[55,56]$. With this method, spatial memory performances and various exploratory behaviors could be assessed quickly [57]. Our previous studies have also shown that when the MrD was stimulated by KA [34], the c-Fos protein was 
expressed in various parts of the limbic system [58] including hippocampus, dentate gyrus, amygdala, and the forebrain cortex. These results indicated the functional connection between the $\mathrm{MrD}$ and the limbic system. In addition, we have also observed the structural connections between the $\mathrm{MrD}$ and some areas of the limbic system [24, 35, 59-61]. In the present study, after KA injection to the $\mathrm{MrD}$, anterograde degenerating axon terminals were found in the synapses of cholinergic neurons in the NBM under the electron microscope. No differences among learning and memory scores in rats of different treatment groups were found before stereotactic injections in the MrD or NBM, but the total number of shocks required for the $90 \%$ positive response was increased significantly after bilateral $\mathrm{KA}$ lesions of the $\mathrm{MrD}$ or NBM, and no significant differences were found between the MrD-lesioned and the NBM-lesioned groups. These results suggested that the bilateral lesions of the MrD led to the decrease of learning and memory ability, which supports the proposition that the structural connection between the MrD and the NBM plays a role in the learning and memory function.

The brain constitutes about $2 \%$ of the body weight, requires about $17 \%$ of the normal cardiac output, and consumes about $20 \%$ of the oxygen utilized by the entire body. The striatum is located in the central position of the brain which is supplied by branches of lenticulostriate arteries arising directly from the proximal portion of the middle cerebral artery [62]. Thus, the ventral striatum derives its blood and oxygen supply more quickly and directly from the heart in comparison to other portions of the brain, and probably carries out important functions of the brain including learning and memory. Based on the findings in the present study and other observations [14, $27,28,35]$, the afferent and efferent signals could be transported from the $\mathrm{MrD}$ to all its connected areas. For instance, the efferent striatonigral fibers from the MrD terminated at the caudal border of the substantia nigra, the pars reticulate [60]. The afferent fibers from the amygdaloid nucleus and the bed nucleus of the stria terminated to the $\operatorname{MrD}[35$, 61]. The MrD and the hippocampus have been proposed to have different roles in learning and memory [42], partly based on the finding on the new pathways among the hippocampus, the amygdala, and the ventromedial region of the striatum in rats [43]. The results obtained from the present study with tract tracing and immunoelectronic microscopy showed a new pathway where neurons of the MrD projected to the NBM and their axon terminals had synaptic connections with the cholinergic neurons of the NBM. Lesion of the new pathway from the $\mathrm{MrD}$ to the $\mathrm{NBM}$ in rats resulted in severe learning and memory deficiency in behavioral tests. Our results also provided evidence to support the proposition that the MrD of the ventral striatum may also join the neural circuitries of the NBM in processing learning and memory signals. Since the cholinergic neurons in the basal forebrain complex are among the first group of neurons known to become degenerated in Alzheimer's disease, the role of the new pathway between the
$\mathrm{MrD}$ and the NBM cholinergic neurons in learning and memory may provide further insights into the pathological mechanism of Alzheimer's disease.

\section{Conclusions}

The results of this study demonstrated a new neural pathway from the $\mathrm{MrD}$ of the ventral striatum to the $\mathrm{NBM}$ at the cellular and subcellular levels and its role in learning and memory as revealed by WGA-HRP tract tracing, histochemical staining, immunoelectronic microscopy, and Y-maze behavioral test after lesions of the MrD. Following WGA-HRP injection into the NBM, positive stained dark-blue particles of WGA-HRP were retrogradely transported to label fusiform neurons in the $\mathrm{MrD}$. The WGA-HRP-labeled terminals of axons from the MrD made synaptic connections with cholinergic neurons in the NBM as demonstrated by WGA-HRP anterograde tract tracing and immunoelectronic microscopy. These results showed fusiform neurons of the $\mathrm{MrD}$, some of them were cholinergic neurons, projected to the NBM and their axon terminals had synaptic connections with the cholinergic neurons of the NBM (Fig. 9). The rats with bilateral MrDs lesions showed degenerated axon terminals in synapses of the cholinergic neurons in the NBM of the rat brain under an electronic microscope and severe learning and memory deficiency of rats in the Y-maze test. The results suggested that the new neural pathway (some of them were cholinergic) from the $\mathrm{MrD}$ to the NBM played an important role in learning and memory. This new pathway might regulate the excitability of the NBM in the cognitive processes and probably might be involved in the complex memory networks of the brain. The new cholinergic neural pathway between the $\mathrm{MrD}$ and the NBM is also proposed to be potentially involved in the pathological mechanism of Alzheimer's disease.

Acknowledgements We thank Mr. Fa Deng for his assistance to improve the figures.

Funding Information This work was supported by the National Natural Foundation of China (NSFC 81271524, NSFC 81371514).

Open Access This article is distributed under the terms of the Creative Commons Attribution 4.0 International License (http:// creativecommons.org/licenses/by/4.0/), which permits unrestricted use, distribution, and reproduction in any medium, provided you give appropriate credit to the original author(s) and the source, provide a link to the Creative Commons license, and indicate if changes were made.

\section{References}

1. Florio TM, Confalone G, Sciarra A, Sotgiu A, Alecci M (2013) Switching ability of over trained movements in a Parkinson's disease rat model. Behav Brain Res 250:326-333 
2. Miyoshi E, Wietzikoski EC, Bortolanza M, Boschen SL, Canteras NS, Izquierdo I, da Cunha C (2012) Both the dorsal hippocampus and the dorsolateral striatum are needed for rat navigation in the Morris water maze. Behav Brain Res 226:171-178

3. Haber SN, Kim K, Mailly P, Calzavara R (2006) Reward-related cortical inputs define a large striatal region in primates that interface with associative cortical connections, providing a substrate for incentive-based learning. J Neurosci 26:8368-8376

4. Yin H, Knowlton BJ (2006) The role of the basal ganglia in habit formation. Nat Rev Neurosci 7:464-476

5. Brasted PJ, Humby T, Dunnett SB, Robbins TW (1997) Unilateral lesions of the dorsal striatum in rats disrupt responding in egocentric space. J Neurosci 17:8919-8926

6. Squire LR, Knowlton B, Musen G (1993) The structure and organization of memory. Annu Rev Psychol 44:453-495

7. Williams ZM, Eskandar EN (2006) Selective enhancement of associative learning by microstimulation of the anterior caudate. Nat Neurosci 9:562-568

8. Oyanagi K, Takahashi H, Wakabayashi K, Ikuta F (1989) Correlative decrease of large neurons in the neostriatum and basal nucleus of Meynert in Alzheimer's disease. Brain Res 504:354-357

9. Albouy G, King BR, Maquet P, Doyon J (2013) Hippocampus and striatum: dynamics and interaction during acquisition and sleep-related motor sequence memory consolidation. Hippocampus 23:985-1004

10. Hallock HL, Arreola AC, Shaw CL, Griffin AL (2013) Dissociable roles of the dorsal striatum and dorsal hippocampus in conditional discrimination and spatial alternation T-maze tasks. Neurobiol Learn Mem 100:108-116

11. Fidalgo C, Conejo NM, Gonzalez-Pardo H, Arias JL (2012) Functional interaction between the dorsal hippocampus and the striatum in visual discrimination learning. J Neurosci Res 90:715-720

12. Woolley DG, Laeremans A, Gantois I, Mantini D, Vermaercke B, op de Beeck HP, Swinnen SP, Wenderoth N et al (2013) Homologous involvement of striatum and prefrontal cortex in rodent and human water maze learning. Proc Natl Acad Sci U S A 110:3131-3136

13. Bradfield LA, Bertran-Gonzalez J, Chieng B, Balleine BW (2013) The thalamostriatal pathway and cholinergic control of goaldirected action: interlacing new with existing learning in the striatum. Neuron 79:153-166

14. Watanabe N, Sakagami M, Haruno M (2013) Reward prediction error signal enhanced by striatum-amygdala interaction explains the acceleration of probabilistic reward learning by emotion. J Neurosci 33:4487-4493

15. Beeman CL, Bauer PS, Pierson JL, Quinn JJ (2013) Hippocampus and medial prefrontal cortex contributions to trace and contextual fear memory expression over time. Learn Mem 20:336-343

16. Vertes RP, Hoover WB, Szigeti-Buck K, Leranth C (2007) Nucleus reuniens of the midline thalamus: link between the medial prefrontal cortex and the hippocampus. Brain Res Bull 71:601-609

17. Cardoso-Cruz H, Sousa M, Vieira JB, Lima D, Galhardo V (2013) Prefrontal cortex and mediodorsal thalamus reduced connectivity is associated with spatial working memory impairment in rats with inflammatory pain. Pain 154:2397-2406

18. Markham CM, Taylor SL, Huhman KL (2010) Role of amygdala and hippocampus in the neural circuit subserving conditioned defeat in Syrian hamsters. Learn Mem 17:109-116

19. Soares JC, Oliveira MG, Ferreira TL (2013) Inactivation of muscarinic receptors impairs place and response learning: implications for multiple memory systems. Neuropharmacology 73C:320-326

20. Zhu L, Wu L, Yu B, Liu X (2011) The participation of a neurocircuit from the paraventricular thalamus to amygdala in the depressive like behavior. Neurosci Lett 488:81-86

21. Ciamei A, Morton AJ (2008) Rigidity in social and emotional memory in the R6/2 mouse model of Huntington's disease. Neurobiol Learn Mem 89:533-544
22. Ciamei A, Morton AJ (2009) Progressive imbalance in the interaction between spatial and procedural memory systems in the R6/2 mouse model of Huntington's disease. Neurobiol Learn Mem 92:417-428

23. Shu SY, Peterson GR, Penny R (1988) The 'marginal division': a new subdivision in the neostriatum of rat. J Chem Neuroanat 1:147-163

24. Shu SY, Bao XM, Zhang C, Li SX, Chan WY, Yew D (2000) A new subdivision, marginal division, in the neostriatum of the monkey brain. Neurochem Res 25:231-237

25. Shu SY, Wu YM, Bao XM, Wen ZB, Huang FH, Li SX, Fu QZ, Ning Q (2002) A new area in the human brain associated with learning and memory: immunohistochemical and functional MRI analysis. Mol Psychiatry 7:1018-1022

26. Shu SY, McGinty JF, Peterson GM (1990) High density zinccontaining and dynorphin B-and, substance P-immunoreactive terminals in the marginal division of the rat striatum. Brain Res Bull 24:201-205

27. Schoen SW, Graybiel AM (1993) Species-specific patterns of glycoprotein expression in the developing rodent caudoputamen: association of $5^{\prime}$-nucleotidase activity with dopamine islands and striosomes in rat, but with extrastriosomal matrix in mouse. J Comp Neurol 333:578-596

28. Talley EM (1996) Distribution of alpha 2A-adrenergic receptor-like immunoreactivity in the rat central nervous system. J Comp Neurol 372:111-134

29. Lavoie B, Parent A (1994) Pedunculopontine nucleus in the squirrel monkey: projections to the basal ganglia as revealed by anterograde tract-tracing methods. J Comp Neurol 344:210-231

30. Shammah-Lagnado SJ, Alheid GF, Heimer L (1999) Afferent connections of the interstitial nucleus of the posterior limb of the anterior commissure and adjacent amygdalostriatal transition area in the rat. Neuroscience 94:1097-1123

31. Heimer L, Zahm DS, Alheid GF (1995) Basal ganglia. In: Paxinos G (ed) The rat nervous system. Academic Press, New York, pp. 579-628

32. Shu SY, Bao X, Li S, Niu D, Xu Z (1999) A new subdivision of mammalian neostriatum with functional implications to learning and memory. J Neurosci Res 58:242-253

33. Chudler EH, Sugiyama K, Dong WK (1993) Nociceptive responses in the neostriatum and globus pallidus of the anesthetized rat. J Neurophysiol 69:1890-1903

34. Bao X, Shu SY, Zhang Y, Zhou G (1997) Expression of c-Fos oncogene protein in the brain following kainic acid injection into the marginal division of the rat striatum. Chin J Neuroanat 13:343-350

35. Ning Q, Shu SY, Bao X, Li Y (2003) Function and fiber connection between amygdala and marginal division of striatum. Chin $\mathrm{J}$ Neuroanat 6:002

36. Li Y, Shu SY, Bao X, Wu W (2000) The connection among the marginal division of striatum, amygdaloid nucleus and bed nucleus of the stria terminalis in the rat brain as revealed by immunohistochemistry. Chin J Neuroanat 16:265-268

37. Shu SY (2003) Marginal division of neostriatum: a subcortical memory center. J Biomed Sci 10:14-29

38. Liu X, Shu SY, Zeng CC, Cai YF, Zhang KH, Wang CX, Fang J (2011) The role of substance $P$ in the marginal division of the neostriatum in learning and memory is mediated through the neurokinin 1receptor in rats. Neurochem Res 36:1896-1902

39. Sadeh T, Shohamy D, Levy DR, Reggev N, Maril A (2011) Cooperation between the hippocampus and the striatum during episodic encoding. J Cogn Neurosci 23:1597-1608

40. Wang C, Shu S, Guo Z, Cai Y, Bao X, Zeng C, Wu B, Hu Z et al (2011) Immunohistochemical localization of mu opioid receptor in the marginal division with comparison to patches in the neostriatum of the rat brain. J Biomed Sci 18:34-42

41. Shu SY, Qing D, Wang B, Zeng QY, Chen YC, Jin Y, Zeng CC, Bao $\mathrm{R}$ (2013) Comparison of microRNA expression in hippocampus and the marginal division $(\mathrm{MrD})$ of the neostriatum in rats. $\mathrm{J}$ Biomed Sci 20:9 
42. Shu SY, Jiang G, Zeng QY, Wang B, Li H, Ma L, Steinbusch H, Song $\mathrm{C}$ et al (2015) The marginal division of the striatum and hippocampus has different role and mechanism in learning and memory. Mol Neurobiol 51:827-830

43. Wang B, Chen YC, Jiang G, Ning Q, Ma L, Chan WY, Wu S, Zhou GQ et al (2016) New learning and memory related pathways among the hippocampus, the amygdala and the ventromedial region of the striatum in rats bin. J Chem Neuroanat 71:13-19

44. Mesulam MM, Mufson EJ, Levey AI, Wainer BH (1983) Cholinergic innervation of cortex by the basal forebrain: cytochemistry and cortical connections of the septal area, diagonal band nuclei, nucleus basalis (substantia innominata), and hypothalamus in the rhesus monkey. J Comp Neurol 214:170-197

45. Bierer LM, Haroutunian V, Gabriel S, Knott PJ, Carlin LS, Purohit DP, Perl DP, Schmeidler J et al (1995) Neurochemical correlates of dementia severity in Alzheimer's disease: relative importance of the cholinergic deficits. J Neurochem 64:749-760

46. Matsuoka N, Yamazaki M, Yamaguchi I (1995) Changes in brain somatostatin in memory-deficient rats: comparison with cholinergic markers. Neurosci. 66:617-626

47. Smith CG, Beninger RJ, Mallet PE, Jhamandas K, Boegman RJ (1994) Basal forebrain injections of the benzodiazepine partial inverse agonist FG 7142 enhance memory of rats in the double Ymaze. Brain Res 666:61-67

48. Connor DJ, Langlais PJ, Thal LJ (1991) Behavioral impairments after lesions of the nucleus basalis by ibotenic acid and quisqualic acid. Brain Res 555:84-90

49. Kesner RP, Crutcher KA, Omana H (1990) Memory deficits following nucleus basalis magnocellularis lesions may be mediated through limbic, but not neocortical, targets. Neurosci. 38:93-102

50. Mesulam MM (1978) Tetramethyl benzidine for horseradish peroxidase neurohistochemistry: a non-carcinogenic blue reaction product with superior sensitivity for visualizing neural afferents and efferents. J Histochem Cytochem 26:106-117

51. Hedreen JC, Bacon SJ, Price DL (1985) A modified histochemical technique to visualize acetylcholinesterase-containing axons. J Histochem Cytochem 33:134-140
52. Carson KA, Mesulam MM (1982) Electron microscopic demonstration of neural connections using horseradish peroxidase: a comparison of the tetramethylbenzidine procedure with seven other histochemical methods. J Histochem Cytochem 30:425-435

53. Paxinos G, Franklin KBJ (2012) The mouse brain in stereotaxic coordinates, 4th edn. Academic Press, Cambridge, MA

54. Stanes MD, Brown CP (1976) Effect of physostigmine on Y-maze discrimination retention in the rat. Psychopharmacologia 46:269276

55. Scoville WB, Milner B (1957) Loss of recent memory after bilateral hippocampal lesions. J Neurol Neurosurg Psychiatry 20:11-21

56. Bao X, Shu SY, Niu D, Xu Z (1998) Distribution of substance P, Leu-enkephalin, neuropeptide Y, and NADPH-d reactivity in the marginal division of cat striatum. Chin J Neurosci 14:213-217

57. Conrad CD, Lupien SJ, Thanasoulis LC, McEwen BS (1997) The effects of type I and type II corticosteroid receptor agonists on exploratory behavior and spatial memory in the Y-maze. Brain Res 759:76-83

58. Maclean PD (1952) Some psychiatric implications of physiological studies on frontotemporal portion of limbic system (visceral brain). Electroencephalogr Clin Neurophysiol 4:407-418

59. Bao X, Shu SY, Li S, Xu Z (1993) The afferent projections of the marginal division in the rat striatum: WGA-HRP retrograde and anterograde transport study. Chin J Neuroanat 9:93-96

60. Shu SY, Bao X, Li S, Xu Z (1993) A immunohistochemical study on the afferent projections of the marginal division of the rat striatum. J Anat 16:509-512

61. Li Y, Shu SY, Bao X, Wu W (1999) Distribution and fiber connection of calcitonin gene-related peptide immunoreactivity in the marginal division of the rat striatum. J First Mil Med Univ 19:201-204

62. Capenter MB, Sutin J (1981) Blood supply of the central nervous system. In: Human Neuroanatomy. Williams \& Wilkings, Baltimore, p. 707

Publisher's Note Springer Nature remains neutral with regard to jurisdictional claims in published maps and institutional affiliations.

\section{Affiliations}

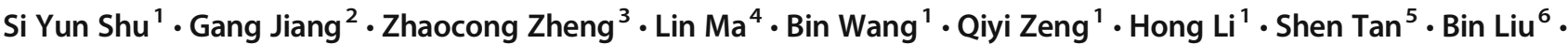 Wood Yee Chan ${ }^{7}$. Sheng $\mathrm{Wu}^{8} \cdot$ Chunhua Zhu $^{8} \cdot{\text { Changke } \mathrm{Li}^{9}}^{9}$ Peng Wang ${ }^{10} \cdot$ Jang-Yen Wu $^{11}$ (D)}

1 Pediatric Center, Zhujiang Hospital of the Southern Medical University, A- 3103, Building 39, No. 253 Gong-ye Road, Haizhu District, Guangzhou 510280, Guangdong, China

2 Department of Ear, Nose and Throat, Zhujiang Hospital of the Southern Medical University, Guangzhou 510282, Guangdong, China

3 Department of Neurosurgery, Fuzhou Central Hospital of Nanjing Military Region, Fuzhou 350025, Fujian, China

4 Department of Radiology, General Hospital of People's Liberation Army, Beijing 100853, China

5 Department of Neurology, Zhujiang Hospital of Southern Medical University, Guangzhou 510282, Guangdong, China
6 Emergency Department, Zhujiang Hospital of Southern Medical University, Guangzhou 510282, Guangdong, China

7 School of Biomedical Sciences, Faculty of Medicine, Chinese University of Hong Kong, Shatin, Hong Kong

8 Nanjing Junqu Hangzhou Sanatorium, 5 Long-jin Road, Hangzhou 310007, China

9 Anesthesia Department of Yue-Bei People Hospital, Shaoguan City 512026, Guangdong, China

10 The Fifth Affiliated Hospital of Southern Medical University, Guangzhou 510900, China

11 Department of Biomedical Science, Charles E. Schmidt College of Medicine, Florida Atlantic University, 777 Glades Road, Boca Raton, FL 33431, USA 